\title{
1 Immunogenicity of novel mRNA COVID-19 vaccine MRT5500 in mice and
}

2 non-human primates

3 Kirill V. Kalnin ${ }^{1 *}$, Timothy Plitnik ${ }^{5}$, Michael Kishko ${ }^{1}$, Jinrong Zhang ${ }^{1}$, Donghui Zhang ${ }^{2}$, Adrien

4 Beauvais $^{1}$, Natalie G. Anosova ${ }^{1}$, Timothy Tibbitts ${ }^{1}$, Joshua M. DiNapoli ${ }^{1}$, Po-Wei D. Huang ${ }^{1}$,

5 James Huleatt ${ }^{2}$, Deanne Vincent ${ }^{2}$, Katherine Fries ${ }^{2}$, Shrirang Karve ${ }^{3}$, Rebecca Goldman ${ }^{3}$, Hardip

6 Gopani $^{3}$, Anusha Dias ${ }^{3}$, Khang Tran $^{3}$, Minnie Zacharia ${ }^{3}$, Xiaobo Gu$^{3}$, Lianne Boeglin ${ }^{3}$, Sudha

7 Chivukula ${ }^{1}$, Ron Swearingen ${ }^{3}$, Victoria Landolfi ${ }^{2}$, Tong-Ming Fu ${ }^{1}$, Frank DeRosa ${ }^{3}$, Danilo

8 Casimiro $^{2}$

9

10

11

12

\section{Summary}

${ }^{1}$ Sanofi Pasteur, 38 Sidney Street, Cambridge, MA 02139, 2Sanofi Pasteur, Discovery Dr., Swiftwater, PA 18370, ${ }^{3}$ Translate Bio, 29 Hartwell Ave, Lexington, MA 02421, ${ }^{4}$ Sanofi Pasteur, 1541 AV Marcel Mérieux, 69280 Marcy l'Etoile, France, ${ }^{5}$ Yoh Services LLC, 38 Sidney Street, Cambridge, MA 02139

An effective vaccine to address the global pandemic of coronavirus disease 2019 (COVID-19) is an urgent public health priority ${ }^{1}$. Novel synthetic mRNA and vector-based vaccine technologies offer an expeditious development path alternative to traditional vaccine approaches. Here we describe the efforts to utilize an mRNA platform for rational design and evaluations of mRNA vaccine candidates based on Spike (S) glycoprotein of Severe Acute Respiratory Syndrome Coronavirus 2 (SARS-CoV-2), the virus causing COVID-19. Several mRNA constructs expressing various structural conformations of S-protein, including wild type (WT), a pre-fusion stabilized mutant (2P), a furin cleavage-site mutant (GSAS) and a double mutant form (2P/GSAS), were tested in a preclinical animal model for their capacity to elicit neutralizing antibodies (nAbs). The lead 2P/GSAS candidate was further assessed in dose-ranging studies in 
24 mice and Cynomolgus macaques. The selected 2P/GSAS vaccine formulation, now designated

25 MRT5500, elicited potent nAbs as measured in two types of neutralization assays. In addition,

26 MRT5500 elicited $\mathrm{T}_{\mathrm{H}} 1$-biased responses in both mouse and non-human primate species, a result

27 that helps to address a hypothetical concern regarding potential vaccine-associated enhanced

28 respiratory diseases associated with $\mathrm{T}_{\mathrm{H}} 2$-biased responses. These data position MRT5500 as a

29 viable vaccine candidate for clinical development against COVID-19.

30 Key words: COVID-19, SARS-CoV-2, vaccine, mRNA, LNP, BALB/c mice, cynomolgus

31 macaques, immunogenicity, neutralization potency, neutralization, microneutralization, ELISA 


\section{Introduction}

33 SARS-CoV-2, previously known as the 2019-novel coronavirus $(2019-\mathrm{nCoV})^{2}$, is a $\beta$ coronavirus with a yet-to-be defined zoonotic origin. The first cases of human infection with severe acute respiratory syndrome (SARS) were reported in December 2019 in China $^{3}$, and later named coronavirus disease 2019 (COVID-19) ${ }^{4}$. In contrast to SARS-CoV-1 virus which caused an outbreak in 2002, SARS-CoV-2 has gained high capacity for human-to-human transmission and quickly spread worldwide. It has caused over 34 million cases of confirmed infection and more than 1,000,000 deaths in 188 countries (https://www.worldometers.info/coronavirus).

An effective vaccine is urgently needed to address this global pandemic.

42 Coronavirus is an enveloped RNA virus, and the viral spike (S) protein on the virion envelope is essential for infection and is the target for host antiviral antibodies ${ }^{5,6}$. The receptor for SARS-

44 CoV-2 is angiotensin-converting enzyme 2 (ACE2), a metalloprotease that also serves as the receptor for SARS-CoV-1 ${ }^{7}$. Most of the COVID-19 vaccine candidates reported are focused on a pre-fusion-stabilized S protein, either as recombinant protein with adjuvant or delivered from

47 viral vectors or as DNA or mRNA vaccines ${ }^{8-15}$. The pre-fusion-stabilized version of SARS-

48 CoV-2 S-protein contains two proline substitutions (2P), at amino acid positions 986 and 987,

49 located near the apex of the central helix and heptad repeat $1^{16}$. Structural studies reveal that the

50 pre-fusion stabilized S closely resembles native S protein on the virion surface; a structure

51 targeted by many reported effective neutralizing antibodies ${ }^{17-19}$. Moreover, the vaccine premises

52 are based on the prior work of MERS-CoV, SARS-CoV and $\mathrm{HCoV}-\mathrm{HKU} 1 \mathrm{~S}$ proteins presented

53 in pre-fusion conformations ${ }^{20-22}$. The ability of S-2P-based vaccines to elicit neutralizing

54 antibodies has been demonstrated ${ }^{8-1023,24}$. 
56 There is a unique feature of SARS-CoV-2 S protein which possesses a polybasic furin cleavage

57 site at the junction of S1 and S2 subunits. This feature is believed to have emerged during viral

58 transmission from zoonotic host to human ${ }^{25-27}$, and is key to SARS-CoV-2 high transmissibility

59 in humans ${ }^{28,29}$. Although robust SARS-CoV-2 infection of human lungs requires a multibasic

60 cleavage $\operatorname{site}^{30}$, interestingly, both cleaved and uncleaved versions of S protein co-exist on

61 virions purified from viral culture on Vero cells ${ }^{31,32}$. Thus, it remains unclear how the cleavage

62 provides an advantage for viral transmission. Also, from a vaccine design perspective, one may

63 speculate that furin cleavage site may result in subtle conformational changes in the trimerized S

64 protein, potentially favoring its interaction with ACE2 ${ }^{26}$.

65

66 These unanswered questions led us to design various forms of S protein constructs involving

67 both $2 \mathrm{P}$ and cleavage site, referred to herein as GSAS mutations. These GSAS constructs were

68 first evaluated for immunogenicity in mice. The 2P/GSAS S mRNA encapsulated in a cationic

69 lipid nanoparticle (LNP) formulation, designated as MRT5500, was subsequently selected for

70 further evaluation. Here we report the results of preclinical evaluation of MRT5500 in mice and

71 non-human primates (NHPs). MRT5500 was administered twice via the intramuscular route (IM)

72 at a three-week interval in both animal models. Results demonstrated that MRT5500 elicited

73 potent neutralizing activity and a $\mathrm{T}_{\mathrm{H}} 1$-biased response in both species. The ability of this vaccine

74 to induce both humoral and cell-mediated antiviral responses identifies MRT5500 as a promising

75 clinical vaccine candidate. 


\section{Results}

\section{Design and selection of mRNA constructs}

78 SARS-CoV-2 S protein, a 1273 amino acid glycoprotein, is expressed and stabilized as a

79 membrane anchored homo-trimer ${ }^{6}$. The receptor binding domain (RBD) has been identified as

80 the critical component to initiate virus attachment to ACE2, a cellular receptor for viral

81 infection ${ }^{33}$. Interestingly, the RBD is present in both up and down configurations in the pre-

82 fusion form of S protein, and the up position has been speculated as the prerequisite for

83 interaction with ACE2 ${ }^{6,31}$. The furin cleavage at the S1/S2 boundary of SARS-CoV-2 S occurs

84 during viral biosynthesis ${ }^{34}$. It is postulated that transition and adaptation to the human host

85 resulted in the acquisition of a furin protease site in the S protein of SARS-CoV-2, which is a

86 unique feature discriminating this virus from SARS-CoV-1 and other SARS-related-CoVs ${ }^{26}$.

87 Approximately $45 \%$ of the total S protein monomers presented within intact SARS-CoV-2

88 virions have been reported as cleaved at the furin cleavage site ${ }^{31}$; however, it is not clear which

89 form is favored by the virus to facilitate the fusion process ${ }^{26,34-36}$.

91 The COVID-19 vaccine hypothesis has been centered around induction of neutralizing

92 antibodies (nAbs) that either block the interaction of the RBD with ACE2, or that prevent the

93 fusion process involving S protein transition from pre- to post-fusion conformation ${ }^{37,38}$. Although

94 the pre-fusion conformation is known to be critical for eliciting a neutralizing response ${ }^{18,19}$, the

95 impact of the furin cleavage site in eliciting neutralizing antibodies requires additional studies.

96 To test the potential contribution of this site, we mutated the furin cleavage site, composed of the

97 polybasic residues RRAR, to GSAS from amino acid position 682 to $685^{30,35}$. Four constructs 
were synthesized as mRNA to represent either wild type (WT), stabilized pre-fusion mutant (2P)

${ }^{20}$, furin cleavage site mutant (GSAS) or a double mutant (2P/GSAS) of SARS-CoV-2 S gene.

These constructs were transfected into a human cell line and their expression levels were verified by Western Blot (Fig. 1a). As expected, endogenous cleavage of WT and 2P constructs, but not GSAS or 2P/GSAS proteins, was observed (Fig. 1a), which yielded a band of approximately 90 $\mathrm{kDa}$ representing $\mathrm{S} 2$.

In order to determine the potential impact of $2 \mathrm{P}$ and GSAS mutations on immunogenicity, we formulated each of the four mRNA constructs within a lipid nanoparticle (LNP), which has been designed for efficient delivery of mRNA vaccines ${ }^{39}$. BALB/c mice were administered two immunizations at a $0.4 \mu \mathrm{g}$ dose of each of four formulations at a three-week interval. Binding antibody activities in the serum samples were assessed via Enzyme-Linked Immunosorbent Assay (ELISA) (Fig. 1b). All four vaccines demonstrated similar levels of binding antibodies 14 days after the first vaccination, and the responses were further enhanced one week after the second dose at day (D) 28. On D35, the IgG geometric mean titers (GMTs) for WT, 2P, GSAS and 2P/GSAS groups were 184343, 200896, 379653 and 201080 respectively. There were no statistically significant differences among those GMT titers.

To understand the potential impact of these mutations on nAbs titers, we tested the ability of immune sera to neutralize the infectivity of GFP reporter pseudoviral particles (RVP) in HEK293 T cells stably over-expressing human ACE2 ${ }^{40}$. RVPs expressed antigenically correct SARS CoV-2 $\mathrm{S}$ protein and GFP reporter genes on lentiviral (HIV) core and were capable of a single round of infection. Pseudoviral neutralization assay $(\mathrm{PsVNa})$ allowed the determination of serum 
121 dilution which can achieve 50\% inhibition of RVP entry (ID 50 ; see Materials and Methods).

122 Contrary to binding antibodies which could be detected at D14 after the first immunization, the

123 neutralizing antibody response could only be detected after the second immunization. Also

124 noted, the spread of the nAb titers within each group were more pronounced when compared to

125 binding antibody titers, with 95\% confidence intervals overlapping each other. On D35, the

126 GMTs for pseudoviral (PsV) nAb titers were 152 for WT, 195 for 2P, 1005 for GSAS and 354

127 for $2 \mathrm{P} / \mathrm{GSAS}$. The neutralizing potential of the GSAS variant was trending slightly higher than

128 2P/GSAS.

129

Another important observation is that ELISA titers were not consistently predictive of

131 neutralizing titers by PsVNa. Some mice in the WT and 2P groups did not seroconvert in the

132 neutralization assay but their endpoint ELISA titers were comparable to the other animals in the

133 group which demonstrated neutralizing activity. We therefore placed greater emphasis on Ps VNa

134 titer for continuing candidate evaluation. Considering the trend towards higher PsVNa observed

135 for the GSAS constructs as well as the expected importance of the pre-fusion conformation, we

136 selected the double mutant 2P/GSAS/LNP formulation, referred to as MRT5500, for further

137 preclinical evaluations.

\section{Serological evaluations of MRT5500 in mice and NHPs}

140 The selected MRT5500 formulation was evaluated in both mouse and NHP studies with a range

141 of doses covering more than 10-fold titration. The hypothesis for this study is that S-specific

142 antibodies blocking viral infection are key for protection, and our evaluation therefore focused

143 on serological responses against SARS-CoV-2 S, with a particular emphasis on neutralizing 
144 titers post vaccination ${ }^{8,9,41}$. Four dose levels in mice were assessed, ranging from 0.2 to $10 \mu \mathrm{g}$

145 per dose. As expected, MRT5500 induced dose-dependent S-specific binding antibodies and

146 neutralizing antibodies in mice (Fig. 2). PsVNa titers were detected in the higher dose groups (5

$147 \mu \mathrm{g}, 10 \mu \mathrm{g})$ after one vaccination, within the titers being more pronounced after the second

148 vaccination at D21 (Fig. 2b). The PsVNa GMTs were 534, 5232, 9370 and 7472 at D35 for the

$1490.2,1.0,5.0$ and $10.0 \mu \mathrm{g}$ dose groups, respectively. There were no statistically significant

150 differences in PsV neutralization titers on D35 between 1, 5 and $10 \mu \mathrm{g}$ groups (Suppl. Table 2),

151 suggesting a dose-saturation effect beyond $1 \mu \mathrm{g}$ in mice. We also demonstrated that the peak

152 PsV titers (D35) in mice were significantly different from the titers observed in a panel of 93

153 convalescent sera from COVID-19 patients (Suppl. Fig. 4).

In NHPs, we evaluated three dose levels: 15, 45 or $135 \mu \mathrm{g}$ per dose. After the first immunization, 156 nearly all NHPs (10 out of 12) developed antibodies reactive to recombinant S protein in ELISA, 157 and the titers were further enhanced after a second immunization at D35 (Suppl. Fig.1) with all 158 NHPs demonstrating high titers of nAbs. The neutralization potency was assessed by two 159 methods: PsVNa (Fig. 2a) and microneutralization (MN) assay (Fig. 2b). In both assays, a dose160 dependent increase in neutralization titer was observed, with GMTs on D35 of 924 for $15 \mu \mathrm{g}$, 161961 for $45 \mu \mathrm{g}$, and 2871 for $135 \mu \mathrm{g}$ in PsVNa. The MN GMTs followed a similar trend, with 162 titers of 555 for $15 \mu \mathrm{g}, 719$ for $45 \mu \mathrm{g}$ and 1877 for the $135 \mu \mathrm{g}$ group. Despite the observed trend 163 towards higher titers with increasing dose, the differences between groups was not statistically 164 significant for either MN or PsV neutralization titers. 
Although we have used two assays to measure the neutralizing potency, the results from both assays were highly correlated (Suppl. Fig. 3 and Suppl. Table 1). Regardless of the dose level tested, D35 PsV and MN titers were approximately 130-fold higher than those of pre-immune animals. Furthermore, the observed PsV and MN titers were significantly higher from titers observed in a panel of 93 convalescent sera from COVID-19 patients (Suppl. Fig. 5).

\section{T-cell profiles of the selected mRNA formulation in NHPs}

Vaccine associated enhanced respiratory disease (VAERD) has been a safety concern for COVID-19 vaccines in development, although the concern at this stage is only a theoretical one ${ }^{1}$. This phenomenon has been reported for whole-inactivated virus vaccines against measles and respiratory syncytial virus (RSV), which were tested in the 1960 s (cit by ${ }^{1}$ ), and one of the disease hypotheses implicates the biased production of $\mathrm{T}_{\mathrm{H}} 2$ cytokines (IL-4, IL-5, IL-13) by antigen-specific CD4 T cells. A similar association between a $\mathrm{T}_{\mathrm{H}} 2$ profile and disease enhancement has been reported for an inactivated SARS-CoV-1 vaccine in mice ${ }^{42}$. Furthermore, less severe cases of SARS were associated with accelerated induction of $\mathrm{T}_{\mathrm{H}} 1$ cell responses ${ }^{43}$, whereas $\mathrm{T}_{\mathrm{H}}$ 2-biased responses have been associated with enhancement of lung disease following infection in mice parenterally vaccinated with inactivated SARS-CoV viral vaccines ${ }^{42,44}$. Similar phenomena have been observed in humans. For example, a SARS-CoV-2-specific cellular response was associated with severity of disease: recovered patients with mild COVID-19 illnesses demonstrated high levels of IFN- $\gamma$ induced by SARS-Cov-2 antigens, while severe pneumonia patients showed significantly lower level of this cytokine ${ }^{45}$. Thus, it is important to understand the T cell profiles induced by MRT5500. 
189 T cell cytokine responses were tested in NHPs three weeks after the second vaccination.

190 Cytokines induced by restimulation with the pooled SARS CoV-2 S protein peptides were 191 assesses in PBMCs on D42 by the IFN- $\gamma$ ( $\mathrm{T}_{\mathrm{H}} 1$ cytokine) and IL-13 ( $\mathrm{T}_{\mathrm{H}} 2$ cytokine) ELISPOT 192 assays. The majority of animals in three dose level groups tested (10 out of 12) demonstrated 193 presence of IFN- $\gamma$ secreting cells, ranging from two to over 100 spot-forming cells per million 194 PBMCs. A dose-response was not observed as the animals in the lower and higher dose level 195 groups showed comparable frequencies of IFN- $\gamma$ secreting cells. In contrast, presence of IL-13 196 cytokine secreting cells was not detected in any of the groups tested and at any dose level, 197 suggesting induction of a $\mathrm{T}_{\mathrm{H}} 1$-biased cellular responses (Fig. 4). These data presented clear 198 evidence for lack of $\mathrm{T}_{\mathrm{H}} 2$ response to $\mathrm{S}$ antigen following vaccination in NHPs.

Similar assessment of cellular immune responses was performed in immune splenocytes in $\mathrm{BALB} / \mathrm{C}$ mice on D35. ELISPOT was conducted in the 5 and $10 \mu \mathrm{g}$ dose groups in Fig. 2. Although BALB/c mice have strong tendency for $\mathrm{T}_{\mathrm{H}} 2$ biased immune responses, following restimulation with the S protein peptide pools splenocytes from the MRT5500 immunized mice secreted predominantly IFNy while IL-5 responses were marginal, suggesting considerable $\mathrm{T}_{\mathrm{H}} 1$ bias (Supplementary Fig.2). Thus, MRT5500 vaccination elicited predominantly $\mathrm{T}_{\mathrm{H}} 1$-biased responses in both animal species.

\section{Discussion}


211 vaccine candidates against SARS-CoV-2. For any vaccine intended to generate antibody-

212 mediated immunity, delivering a conformationally correct protein is critical ${ }^{1}$. Lessons have been

213 learned from RSV F protein where the post-fusion form elicited poor neutralizing antibodies,

214 albeit extremely immunogenic in humans ${ }^{46}$, and the post-fusion form $\mathrm{F}$ antigen vaccine has

215 failed to provide any protection against RSV infection ${ }^{47}$. Thus, our focus in this study was to

216 identify mutations that could stabilize the pre-fusion form of S antigen upon expression. In

217 contrast to the other $\mathrm{S}$ antigen mRNA vaccines under evaluations $9,10,23,24,48$, we have incorporated

218 a unique mutation at cleavage site GSAS, in addition to $2 \mathrm{P}$, which has enhanced features to lock

219 the S protein in the pre-fusion form. There were two considerations for this design. First, it is not

220 known whether 2P mutations alone, located at the apex of the central helix and heptad repeat 1,

221 are sufficient for locking the S antigen in the pre-fusion form. Second, it has been hypothesized

222 that cleavage of S antigen into S1 and S2 subunits is part of the transition from the pre-fusion to

223 post-fusion form during viral entry ${ }^{19}$. Thus, by blocking the furin cleavage site, we have added

224 another layer for prevention of pre-fusion to post-fusion conversion.

226 The two GSAS containing mutants (GSAS and double mutant 2P/GSAS) resulted in nAb titers

227 that trended higher than the WT and 2P analogues (Fig. 1c). While the nAb levels from these

228 two GSAS-containing antigens were not significantly different from one another, we believe

229 there could be two explanations for this: first, the mutation on the furin cleavage site may alter S

230 protein trafficking efficiency to the cell surface. Furin could be active in the trans-golgi network,

231 cell surface or endosome in processing viral glycoproteins during viral maturation ${ }^{49}$. Thus, it is

232 possible that blocking furin cleavage may have changed S protein trafficking from the ER to the

233 cell surface. Although this hypothesis is unlikely, MERS and SARS, as well as other coronavirus 
234 S protein, have been reported to be missing a furin cleavage site, indicating that furin cleavage is

235 not absolutely necessary for viral maturation ${ }^{26,27,30,35}$. Nonetheless, additional investigation

236 would be needed to further understand the effect of the furin cleavage site on viral

237 morphogenesis and SARS-CoV-2 S protein trafficking. A second possibility, which is more

238 likely, is that we tested the mRNA vaccines at a poorly differentiating dose level $(0.4 \mu \mathrm{g} / \mathrm{dose})$ in

239 mice (Fig. 1c). Our results in a subsequent experiment (Fig. 2) confirmed that the saturation

240 point for neutralizing antibody responses in mice was between $0.2 \mu \mathrm{g}$ and $1 \mu \mathrm{g}$ per dose. With

241 these considerations, we selected the double mutant formulation, MRT5500, to favor the pre-

242 fusion form. Our dose ranging studies in NHPs confirmed the potency of MRT5500 in eliciting

243 neutralizing antibodies. Although the sample size of our experiment (4 animals per group) was

244 not enough to discriminate between the dose regimens, it suggested the potential of MRT5500

245 vaccine candidate to elicit potent neutralizing antibodies in clinic.

247 The long-term durability of our vaccine candidates for COVID-19 across all modalities is still

248 under investigation. As a novel vaccine platform, mRNA can drive efficient de novo antigen

249 expression, which is expected to activate immune responses. However, it is unknown whether

250 the transient nature of antigen expression is sufficient in driving adequate germinal center

251 formation which is needed for effective expansion and maturation of antigen-specific B cells.

252 Although an mRNA vaccine for cytomegalovirus $\mathrm{gB}$ has demonstrated sustained antibody

253 responses in rabbits up to 20 weeks $^{50}$, the durability for $\mathrm{S}$ antigen mRNA remains an important

254 focus for COVID-19 vaccine research. It should be noted that natural infection in COVID-19

255 patients, especially those of mild and asymptomatic cases, induce antibodies that decay rapidly

256 in convalescent phase, with some drifting down to baseline within three months after diagnosis ${ }^{51}$. 
257 Additional preclinical studies are ongoing to further our understanding and characterization of

258 MRT5500 and its immunological effects for applications towards COVID-19.

259

260 In summary, we have utilized mRNA technology for the rapid evaluation of vaccine candidates

261 for COVID-19, and our results led to the selection of a double mutant candidate which has a

262 better potential to preserve a pre-fusion conformation. The candidate MRT5500 has been shown

263 to be immunogenic by eliciting potent neutralizing antibodies in mice and NHPs, and $\mathrm{T}_{\mathrm{H}} 1$-biased

264 cellular immune responses. The candidate is positioned for further development in clinical

265 studies as a vaccine for the prevention of COVID-19. 


\section{Acknowledgements}

267 We are grateful for assistance on statistical analysis by Alice Raillard and Nada Assi of Sanofi

268 Pasteur. We also want to thank exceptional support from veterinary staff and animal research

269 staff at New Iberia Research Center, LA and Covance, Denver, PA. The research is funded by

270 Translate Bio and Sanofi Pasteur. 


\section{Material and methods}

\section{2 mRNA synthesis, lipid nanoparticle formulation and expression assay}

273 Messenger RNA incorporating coding sequences containing either the wild type (WT) sequence, 274 stabilized pre-fusion mutant $(2 \mathrm{P})^{52}$, furin cleavage site mutant (GSAS) ${ }^{35,53}$ or double mutant (2P, 275 GSAS) of the full length SARS-CoV-2 spike glycoprotein were synthesized by in vitro 276 transcription employing RNA polymerase with a plasmid DNA template encoding the desired 277 gene using unmodified nucleotides. The resulting purified precursor mRNA was reacted further 278 via enzymatic addition of a 5' cap structure (Cap 1) and a 3' poly(A) tail of approximately 200 279 nucleotides in length as determined by gel electrophoresis. The vaccine sequence is based on 280 Wuhan Hu-1 strain (Genbank accession MN908947). Preparation of mRNA/lipid nanoparticle 281 (LNP) formulations was described previously ${ }^{54}$. Briefly, an ethanolic solution of a mixture of 282 lipids (ionizable lipid, phosphatidylethanolamine, cholesterol and polyethylene glycol-lipid) at a 283 fixed lipid and mRNA ratio were combined with an aqueous buffered solution of target mRNA 284 at an acidic $\mathrm{pH}$ under controlled conditions to yield a suspension of uniform LNPs. Upon 285 ultrafiltration and diafiltration into a suitable diluent system, the resulting nanoparticle 286 suspensions were diluted to final concentration, filtered and stored frozen at $-80^{\circ} \mathrm{C}$ until use.

287 Expression of S-proteins from cells transfected with synthetic mRNAs was evaluated by Western 288 blot. Briefly, 5 X10 $10^{5}$ HEK293 cells were transfected using $1 \mu \mathrm{g}$ of mRNA complexed with 289 Lipofectamine 2000 , and allowed to incubate $20 \mathrm{hs}$ at $37^{\circ} \mathrm{C}$. Cells were harvested after incubation period, and lysates were analyzed by Western Blot as described elsewhere ${ }^{55}$.

\section{Animal studies}


Animal experiments were carried out in compliance with all pertinent US National Institutes of Health regulations and were conducted with approved animal protocols from the Institutional Animal Care and Use Committee (IACUC) at the research facilities.

296 The mouse studies were conducted at Covance Inc, Denver, PA. Female specific pathogen free

$297 \mathrm{BALB} / \mathrm{c}$ mice of 6-8-week-old were vaccinated in groups of 10, with $50 \mu \mathrm{L}$ of the designated 298 mRNA/LNP formulation into one hind leg for the prime (D0) and the contralateral hind leg for 299 the boost (D21). Sera were collected on D-7, 14, 21, 28 and 35 from the orbital sinus or by exsanguination on D35 by the jugular vein/carotid artery. For cell-mediated response measurements, splenocytes from mice were collected on D35.

302 Cynomolgus macaques of Mauritian origin 2-6 years of age and weighing in a range of 2-6 kg 303 were administered with $500 \mu \mathrm{L}$ mRNA/LNP formulations via IM route into the deltoid of the 304 right forelimb for the prime (D0) and the opposite forelimb for the boost (D21). Sera were 305 collected on D-4, 14, 21, 28, 35, 42 and, PBMCs were isolated on D42. All immunizations and blood draws occurred under sedation with Ketamine $\mathrm{HCl}(10 \mathrm{mg} / \mathrm{kg})$ or Telazol (4-8mg/kg IM).

\section{Convalescent human sera}

309 Convalescent serum panel $(\mathrm{N}=93)$ was obtained from commercial vendors (Sanguine Biobank, 310 iSpecimen and PPD). These subjects had a PCR positive diagnosis of COVID-19 and the serum 311 samples were collected within 3 months following diagnosis.

\section{Enzyme-Linked Immunosorbent Assay (ELISA)}


314 Nunc MaxiSorb plates were coated with SARS-CoV S-GCN4 protein (custom made at GeneArt)

315 protein at $0.5 \mu \mathrm{g} / \mathrm{ml}$ in PBS overnight at $4^{\circ} \mathrm{C}$. Plates were washed 3 times with PBS-Tween $0.1 \%$

316 before blocking with $1 \%$ BSA in PBS-Tween $0.1 \%$ for $1 \mathrm{~h}$ at ambient temperature. Samples

317 were plated with 1:450 initial dilution followed by 3-fold, 7-point serial dilution in blocking

318 buffer. Plates were washed 3 times after 1 -h incubation at room temperature before adding $50 \mu \mathrm{l}$

319 of 1:5000 Rabbit anti-human IgG (Jackson Immuno Research) to each well. Plates were

320 incubated at room temperature for $1 \mathrm{hr}$ and washed $3 \mathrm{x}$. Plates were developed using Pierce 1-Step

321 Ultra TMB-ELISA Substrate Solution for $0.1 \mathrm{~h}$ and stopped by TMB stop solution. Plates were

322 read at $450 \mathrm{~nm}$ in SpectraMax plate reader. Antibody titers were reported as the highest dilution

323 that is $\geq 0.2$ Optical Density (OD) cutoff.

324 For mouse sera, the procedure was similar except the following differences. First, 2019-nCoV

325 Spike protein $(\mathrm{S} 1+\mathrm{S} 2)$ ectodomain (Sino Biological, Cat\# 40589-V08B1) was used as substrate

326 and coated at $2 \mu \mathrm{g} / \mathrm{mL}$ concentration in bicarbonate buffer overnight at $4^{\circ} \mathrm{C}$. Second, the plates

327 were developed using colorimetric substrate, Sure Blue TMB 1-component (SERA CARE, KPL

328 Cat\# 5120-0077) and stopped by Stop solution (SERA CARE Sure Blue, KPL Cat\# 5120-0024).

329 The endpoint antibody titer for each sample was determined as the highest dilution which gave

330 OD value $3 x$ higher than the background.

\section{Pseudovirus Neutralization Assay}

333 Serum samples were diluted 1:4 in media (FluoroBrite phenol red free DMEM $+10 \%$ FBS

$334+10 \mathrm{mM}$ HEPES $+1 \%$ PS $+1 \%$ Glutamax) and heat inactivated at $56^{\circ} \mathrm{C}$ for $0.5 \mathrm{~h}$. A further, 2-

335 fold serial dilution of the heat inactivated serum were prepared and mixed with the reporter virus

336 particle (RVP) -GFP (Integral Molecular) diluted to contain 300 infectious particles per well and 
337 incubated for $1 \mathrm{~h}$ at $37^{\circ} \mathrm{C} .96$-well plates of $50 \%$ confluent $293 \mathrm{~T}$-hsACE2 clonal cells in $75 \mu \mathrm{L}$

338 volume were inoculated with $50 \mu \mathrm{L}$ of the serum/virus mixtures and incubated at $37^{\circ} \mathrm{C}$ for $72 \mathrm{~h}$.

339 At the end of the incubation, plates were scanned on a high-content imager and individual GFP

340 expressing cells were counted. The inhibitory dilution titer ( ID $\left._{50}\right)$ was reported as the reciprocal

341 of the dilution that reduced the number of virus plaques in the test by $50 \%$. ID 50 for each test

342 sample was interpolated by calculating the slope and intercept using the last dilution with a

343 plaque number below the $50 \%$ neutralization point and the first dilution with a plaque number

344 above the $50 \%$ neutralization point. $\mathrm{ID}_{50}$ Titer $=(50 \%$ neutralization point - intercept $\left.) / \mathrm{slope}\right)$.

\section{Microneutralization assay}

347 Serial two-fold dilutions of heat inactivated serum samples were incubated with a challenge dose targeting 100 50\% tissue culture infectious dose (TCID $\left.{ }_{50}\right)$ of SARS-CoV-2 (strain USAWA1/2020 [BEI Resources; catalog\# NR-52281]) at $37^{\circ} \mathrm{C}$ with $5 \% \mathrm{CO}_{2}$ for 1 hour (h). The serum-virus mixtures were inoculated into wells of a 96-well microplate with preformed Vero E6 incubated at $37^{\circ} \mathrm{C}$ with $5 \% \mathrm{CO}_{2}$ for 2 days. After washing and fixation of the Vero E6 cell monolayers, SARS-CoV-2 antigen production in cells was detected by successive incubations

355 with an anti-SARS-CoV nucleoprotein mouse monoclonal antibody (Sino Biological catalog\# 40143-MM05), HRP IgG conjugate (Jackson ImmunoResearch Laboratories, catalog \#115-035-

357 062), and a chromogenic substrate. The resulting optical density (OD) was measured using a 358 microplate reader. The reduction in SARS-CoV-2 infectivity, as compared to that in the virus 359 control wells, constitutes a positive neutralization reaction indicating the presence of neutralizing 
360

361

362

363

364

365

366

367

368

369

370

371

372

373

374

375

376

377 million cells was reported.

378 For testing cytokine responses in NHPs Monkey IFNy ELISPOT (CTL, cat\# 3421M-4APW) and

antibodies in the serum sample. The $50 \%$ neutralization titer $\left(\mathrm{MN}_{50}\right)$ was defined as the reciprocal of the serum dilution for which the virus infectivity was reduced by $50 \%$ relative to the virus control on each plate. The $\mathrm{MN}_{50}$ for each sample was interpolated by calculating the slope and intercept using the last dilution with an OD below the 50\% neutralization point and the first dilution with an $\mathrm{OD}$ above the $50 \%$ neutralization point; $\mathrm{MN}_{50}$ Titer $=(\mathrm{OD}$ of $50 \%$ neutralization point - intercept)/slope.

\section{Cytokine ELISPOT analysis}

For testing cytokine responses in mice CTL ELISPOT kits (Mouse IFN- $\gamma /$ IL-5 Double-Color enzymatic ELISPOT, Immunospot) were used according to the manufacture's protocols. Briefly, freshly isolated splenocytes were resuspended in CTL-Test Media and incubated overnight at 300,000 cells per well with commercially available SARS-CoV-2 S peptide pools. PepMix ${ }^{\mathrm{TM}}$ SARS-CoV-2 (Spike Glycoprotein, Cat\# PM-WCPV-S-1, JPT, Germany) peptide pool 1 and pool 2 were used at the final concentration of $2 \mu \mathrm{g} / \mathrm{ml}$ per well. Concanavalin A (CovA, Sigma $\mathrm{C} 5275)$ at concentration of $1 \mu \mathrm{g} / \mathrm{ml}$ was used for a positive control stimulation. After overnight incubation, the plates were washed and developed per manufacturer instructions. Spots were scanned and analyzed by the CTL technical team. The number of cytokines producing cells per IL-13 ELISPOT kits (CTL, cat\# 3470M-4APW) were used. Previously frozen PBMCs were 380 washed, resuspended in culture medium provided by the kit and enumerated. PepMix ${ }^{\mathrm{TM}}$ SARS381 CoV-2 peptide pools as well as CovA were used for stimulation as described above. PBMC were 382 plated at 300,000 cells per well and stimulated overnight. After overnight incubation the plates 
383 were washed and developed per manufacturer instructions. The plates were dried overnight,

384 scanned, and spots were counted using a CTL analyzer (Immunospot S6 Universal Analyzer,

385 CTL). The data were reported as spot forming cells (SFC) per million PBMCs.

\section{Statistical analysis}

387 Data were log-10 transformed prior to statistical analysis. All statistical tests were two-sided, and 388 the nominal level of statistical significance was set to $\alpha=5 \%$. All analyses were performed on

389 SEG SAS v9.4®. Statistical comparisons among different groups (different dose levels or 390 constructs in a particular study) or between D35 and pre-bleed were conducted using mixed 391 effect model for repeated measures, the model included group, day and their interactions, where 392 day was specified as repeated measures.

393 When assessing pairwise correlations among IgG, MN, PsVNa in NHP study, we proposed a 394 two-stage approach to separate the intra-and inter-variabilities for the repeated measures. Stage

395 1: we calculated the correlation coefficient for each individual subject based on observations 396 over time per subject; Stage 2: we then estimated the mean and 95\% CI of group correlation 397 coefficient based on individual coefficient estimates. The analysis was based on log 10 398 transformed data.

399 Statistical comparisons among different groups (i.e. different dose levels) and the convalescent 400 sera on D35 were conducted using either analysis of variance (ANOVA) or Wilcoxon Rank Sum 401 Test. 


\section{Figure and Table Legends}

404

405

406

407

408

409

410

411

412

413

414

415

416

417

418

419

420

421

422

423

424

425

Figure 1: Comparison of S antigen constructs. (a) In vitro expression of S SARS-CoV-2 protein was assessed in Western blot analysis HEK293 cells were transfected with $1 \mu \mathrm{g}$ mRNA construct of control diluent (Mock), wild type (WT), stabilized pre-fusion mutant (2P), cleavage site mutant (GSAS) or double mutant containing both mutations (2P/GSAS) and the respective expressed S proteins were detected with rabbit anti-SARS-CoV spike protein polyclonal antibody (NB100-56578, Novus Biologicals). (b) Serum antibodies reactive to S antigen and (c) serum neutralizing titers in mice immunized with mRNA vaccines. BALB/c female mice $(n=8)$ were immunized at D0 and D21 with $0.4 \mu \mathrm{g}$ of WT, 2P, GSA, 2P/GSAS mRNA vaccine formulations. Sera samples at indicated timepoints were tested for reactivity to recombinant $\mathrm{S}$ protein in ELISA or tested in a pseudovirus neutralization assay. The $50 \%$ inhibitory dilution titers $\left(\mathrm{ID}_{50}\right)$ were calculated as the reciprocal of the dilution that reduced the number of virus plaques in the test by $50 \%$. Each dot represents an individual serum sample and the line represents the geometric mean with standard deviation for the group. the dotted line below for each panel represents the lower limit of assay readout.

Figure 2: Serological evaluation of MRT5500 formulation in mice. Groups of BALB/c mice $(\mathrm{n}=8)$ were immunized at D0 and D21 with $0.2,1.0,5.0$ or $10.0 \mu \mathrm{g}$ dose of MRT5500 formulation. Serum samples at the indicated time were tested in ELISA (a) and PsVNa (b). Each symbol represents a serum sample and the line is the geometric mean with standard deviation of the group. The dotted line in each panel represents the lower limit of assay detection. PsV neutralization titers $(\mathrm{nAb})$ of the human convalescent serum panel $(\mathrm{n}=93)$ were defined in separate experiment and shown in the same scale on Y-axis as other samples. 
427 Figure 3: Neutralizing titers in NHPs vaccinated with MRT5500 formulation. Groups of

428 cynomolgus macaques $(n=4)$ were vaccinated with MRT5500 at 15, 45 or $135 \mu$ ger dose at D0

429 and D21, and serum samples collected at the indicated timepoints were tested in PsVNa (a) and

$430 \mathrm{MN}$ assay (b). Each symbol represents an individual sample and the line geometric means for the

431 group. The neutralization titer of the sample, shown as $\mathrm{ID}_{50}$, was defined as the reciprocal of the

432 highest test serum dilution for which the virus infectivity was reduced by $50 \%$ when compared to

433 the assay challenge virus dose. PsV and MN neutralization titers (NAb) of the human

434 convalescent serum panel $(n=93)$ were defined in separate experiment and shown in the same

435 scale on Y-axis as other samples.

437 Figure 4: Assessment of T-cell responses in NHPs vaccinated with MRT5500. PBMCs collected

438 at D42, 21 days post the second vaccination, were incubated overnight with the SARS-Cov-2 S-

439 protein peptide pools representing the entire $\mathrm{S}$ open reading frame. The frequencies of PBMC

440 secreting IFNy (left panels) or IL-13 (right panels) were calculated as spots forming cells (SFC)

441 per million PBMC. Each symbol represents an individual sample, and the bar represent the 
443 geometric mean for the group. The dotted line represents the lower limit for detection.

\section{Figures}

445 Figure 1
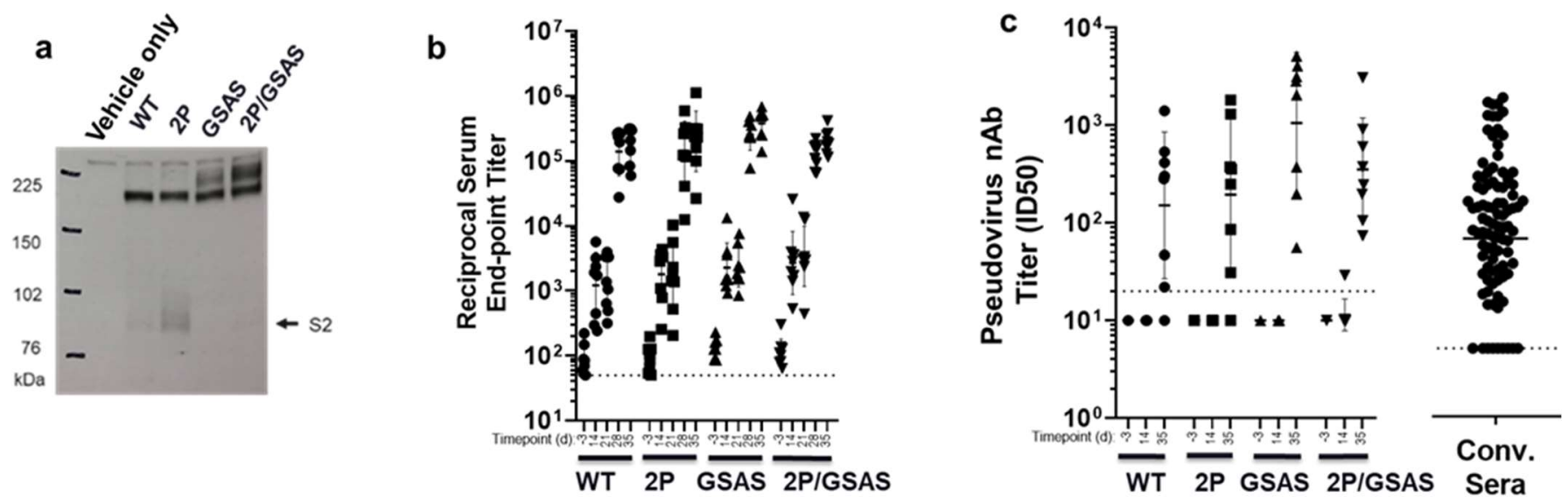

446

Figure 2
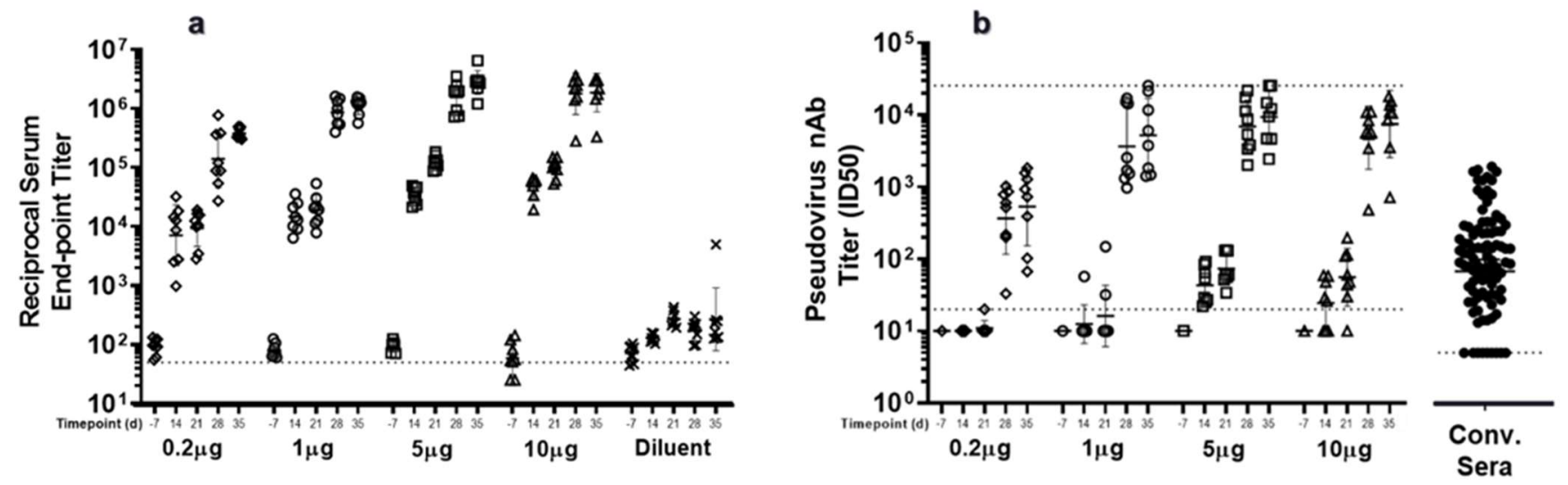

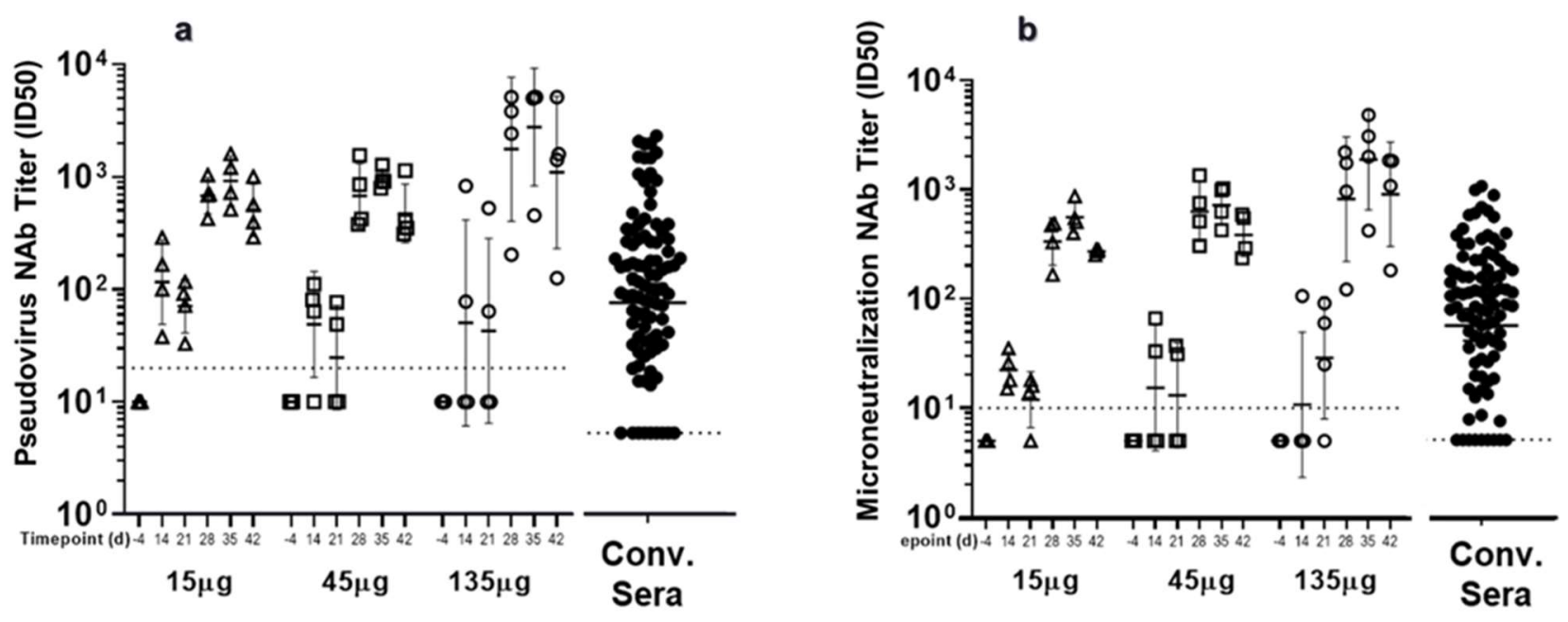

$455 \quad$ Figure 4

\section{Peptide Pool: $\mathbf{S 1}$}

Peptide Pool: S2
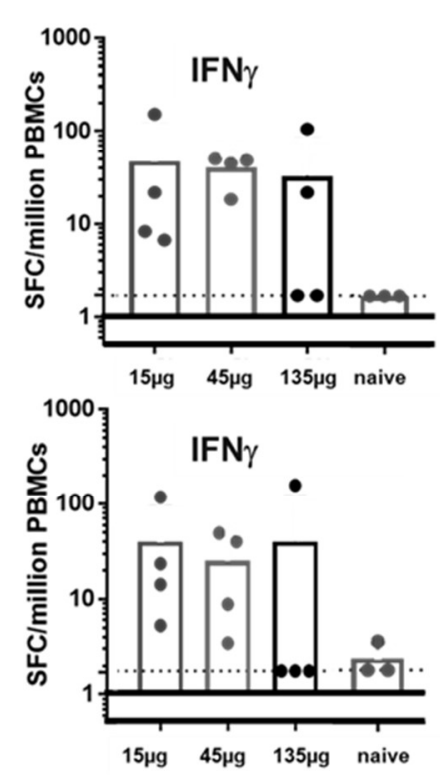
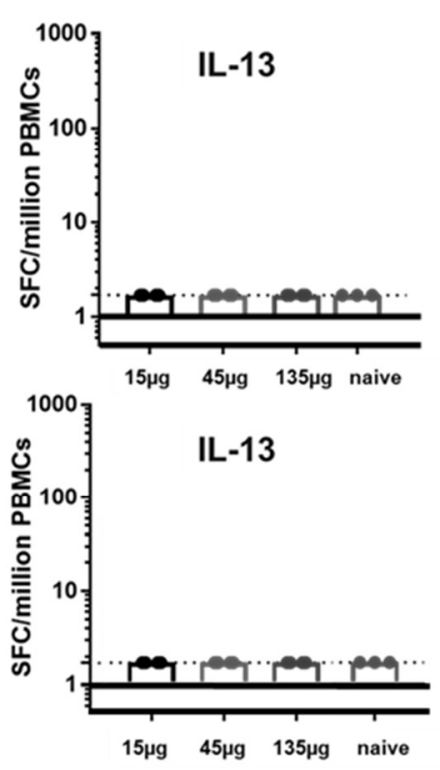


\section{Literature}

461

462

4631 Graham, B. S. Rapid COVID-19 vaccine development. Science 368, 945-946,

464 doi:10.1126/science.abb8923 (2020).

4652 Shi, Y., Wang, N. \& Zou, Q. M. [Progress and challenge of vaccine development against 2019

466

467

468 novel coronavirus (2019-nCoV)]. Zhonghua Yu Fang Yi Xue Za Zhi 54, E029,

469 doi:10.3760/cma.j.cn112150-20200317-00366 (2020).

3 Lu, H., Stratton, C. W. \& Tang, Y. W. Outbreak of pneumonia of unknown etiology in Wuhan, China: The mystery and the miracle. J Med Virol 92, 401-402, doi:10.1002/jmv.25678 (2020). Helmy, Y. A. et al. The COVID-19 Pandemic: A Comprehensive Review of Taxonomy, Genetics,

472 Epidemiology, Diagnosis, Treatment, and Control. J Clin Med 9, doi:10.3390/jcm9041225 (2020).

473

474

475

476

477

478

479

480

481

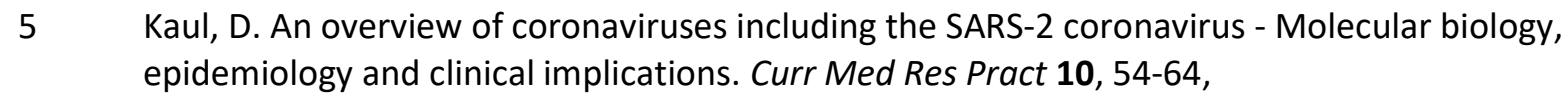
doi:10.1016/j.cmrp.2020.04.001 (2020).

6 Wrapp, D. et al. Cryo-EM structure of the 2019-nCoV spike in the prefusion conformation. Science 367, 1260-1263, doi:10.1126/science.abb2507 (2020).

7 Shang, J. et al. Structural basis of receptor recognition by SARS-CoV-2. Nature 581, 221-224, doi:10.1038/s41586-020-2179-y (2020).

8 Mercado, N. B. et al. Single-shot Ad26 vaccine protects against SARS-CoV-2 in rhesus macaques. Nature, doi:10.1038/s41586-020-2607-z (2020).

482

483

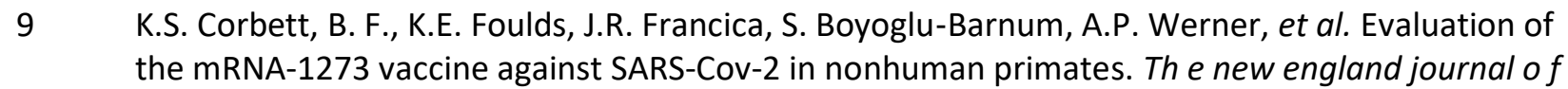
medicine (2020).

10 Corbett, K. e. a. K. S. C., Darin Edwards2\#, Sarah R. Leist3\#, Olubukola M. Abiona1, Seyhan et al. SARS-Cov-2 mRNA Vaccine Development Enabled by Prpototype Pathogen Preparedness. doi:doi: https://doi.org/10.1101/2020.06.11.145920. (2020). Folegatti, P. M., Aley, P. K., Angus, B., Becker, S., Belij-Rammerstorfer, S., Bellamy. Safety and immunogenicity of ChAd0zx1 nCov1-19 vaccine against SARS-Cov-2: a preliminary report of phase $1 / 2$, single-blind, randomised controlled trial. Open access (2020). Gao, Q. et al. Rapid development of an inactivated vaccine candidate for SARS-CoV-2. Science, doi:10.1126/science.abc1932 (2020).

492

493

13 Stefano, M. L., Kream, R. M. \& Stefano, G. B. A Novel Vaccine Employing Non-Replicating Rabies Virus Expressing Chimeric SARS-CoV-2 Spike Protein Domains: Functional Inhibition of Viral/Nicotinic Acetylcholine Receptor Complexes. Med Sci Monit 26, e926016, doi:10.12659/MSM.926016 (2020). $\mathrm{Yu}, \mathrm{J}$. et al. DNA vaccine protection against SARS-CoV-2 in rhesus macaques. Science, doi:10.1126/science.abc6284 (2020). McKay, P. F. et al. Self-amplifying RNA SARS-CoV-2 lipid nanoparticle vaccine candidate induces high neutralizing antibody titers in mice. Nat Commun 11, 3523, doi:10.1038/s41467-02017409-9 (2020). 1501-1505, doi:10.1126/science.abd0826 (2020). 
50317 Joyce, M. G. et al. A Cryptic Site of Vulnerability on the Receptor Binding Domain of the SARSCoV-2 Spike Glycoprotein. bioRxiv, doi:10.1101/2020.03.15.992883 (2020).

18 Huo, J. et al. Neutralization of SARS-CoV-2 by Destruction of the Prefusion Spike. Cell Host Microbe, doi:10.1016/j.chom.2020.06.010 (2020).

19 Cai, Y. et al. Distinct conformational states of SARS-CoV-2 spike protein. Science, doi:10.1126/science.abd4251 (2020).

20 Walls, A. C. et al. Cryo-electron microscopy structure of a coronavirus spike glycoprotein trimer. Nature 531, 114-117, doi:10.1038/nature16988 (2016).

21 Kirchdoerfer, R. N. et al. Pre-fusion structure of a human coronavirus spike protein. Nature 531, 118-121, doi:10.1038/nature17200 (2016).

22 Pallesen, J. et al. Immunogenicity and structures of a rationally designed prefusion MERS-CoV spike antigen. Proc Natl Acad Sci U S A 114, E7348-E7357, doi:10.1073/pnas.1707304114 (2017).

23 Walsh, E. E. et al. RNA-Based COVID-19 Vaccine BNT162b2 Selected for a Pivotal Efficacy Study. medRxiv, doi:10.1101/2020.08.17.20176651 (2020).

24 Jackson, L. A. et al. An mRNA Vaccine against SARS-CoV-2 - Preliminary Report. N Engl J Med, doi:10.1056/NEJMoa2022483 (2020).

25 Xiao, K. et al. Isolation of SARS-CoV-2-related coronavirus from Malayan pangolins. Nature 583, 286-289, doi:10.1038/s41586-020-2313-x (2020).

26 Wrobel, A. G. et al. SARS-CoV-2 and bat RaTG13 spike glycoprotein structures inform on virus evolution and furin-cleavage effects. Nat Struct Mol Biol 27, 763-767, doi:10.1038/s41594-0200468-7 (2020).

27 Wrobel, A. G. et al. Author Correction: SARS-CoV-2 and bat RaTG13 spike glycoprotein structures inform on virus evolution and furin-cleavage effects. Nat Struct Mol Biol, doi:10.1038/s41594020-0509-2 (2020).

28 Lauxmann, M. A., Santucci, N. E. \& Autran-Gomez, A. M. The SARS-CoV-2 Coronavirus and the COVID-19 Outbreak. Int Braz J Urol 46, 6-18, doi:10.1590/S1677-5538.IBJU.2020.S101 (2020).

29 Frutos, R., Serra-Cobo, J., Chen, T. \& Devaux, C. A. COVID-19: Time to exonerate the pangolin from the transmission of SARS-CoV-2 to humans. Infect Genet Evol 84, 104493, doi:10.1016/j.meegid.2020.104493 (2020).

30 Hoffmann, M., Kleine-Weber, H. \& Pohlmann, S. A Multibasic Cleavage Site in the Spike Protein of SARS-CoV-2 Is Essential for Infection of Human Lung Cells. Mol Cell 78, 779-784 e775, doi:10.1016/j.molcel.2020.04.022 (2020).

$31 \mathrm{Ke}, \mathrm{Z}$. et al. Structures and distributions of SARS-CoV-2 spike proteins on intact virions. Nature, doi:10.1038/s41586-020-2665-2 (2020).

32 Peacok, T. P., Goldhill, D.H., Zhou, J., Bailon, L., Frise,R., Swann, O.C., Kugathasan, R., Penn, R., Brown, J.C., Sanchez-David, R.Y., Braga, L., Williamson, M.K., Hassard, J.A., Staller, E., Hanley, B., Osborn, M., Davidson, A.D., Mathews, D.A., Barclay, W.S. . The furin cleavage site of SARS-COV-2 spike protein is a key determinant for transmission due to enhanced replication in airway cells. doi:https://doi.org/10.1101/2020.09.30.318311. (2020).

33 Prabakaran, P., Xiao, X. \& Dimitrov, D. S. A model of the ACE2 structure and function as a SARSCoV receptor. Biochem. Biophys. Res. Commun 314, 235-241 (2004).

34 Walls, A. C. et al. Structure, Function, and Antigenicity of the SARS-CoV-2 Spike Glycoprotein. Cell 181, 281-292 e286, doi:10.1016/j.cell.2020.02.058 (2020).

35 Xing, Y., Li, X., Gao, X. \& Dong, Q. Natural Polymorphisms Are Present in the Furin Cleavage Site of the SARS-CoV-2 Spike Glycoprotein. Front Genet 11, 783, doi:10.3389/fgene.2020.00783 (2020). 
Coutard, B. et al. The spike glycoprotein of the new coronavirus 2019-nCoV contains a furin-like cleavage site absent in CoV of the same clade. Antiviral Res 176, 104742, doi:10.1016/j.antiviral.2020.104742 (2020).

37 Wang, S. et al. Endocytosis of the receptor-binding domain of SARS-CoV spike protein together with virus receptor ACE2. Virus Res 136, 8-15, doi:10.1016/j.virusres.2008.03.004 (2008).

38 Hoffmann, M. et al. SARS-CoV-2 Cell Entry Depends on ACE2 and TMPRSS2 and Is Blocked by a Clinically Proven Protease Inhibitor. Cell 181, 271-280 e278, doi:10.1016/j.cell.2020.02.052 (2020).

39 Reichmuth, A. M., Oberli, M. A., Jaklenec, A., Langer, R. \& Blankschtein, D. mRNA vaccine delivery using lipid nanoparticles. Ther Deliv 7, 319-334, doi:10.4155/tde-2016-0006 (2016).

40 Crawford, K. H. D. et al. Protocol and Reagents for Pseudotyping Lentiviral Particles with SARSCoV-2 Spike Protein for Neutralization Assays. Viruses 12, doi:10.3390/v12050513 (2020).

41 Jingyou Yu1*, L. H. T., Lauren Peter1*, Noe B. Mercado1*, Katherine McMahan1*, et al. DNA Vaccine Protection Against SARS-CoV-2 in Rhesus Macaques. (2020).

42 Bolles, M. et al. A double-inactivated severe acute respiratory syndrome coronavirus vaccine provides incomplete protection in mice and induces increased eosinophilic proinflammatory pulmonary response upon challenge. J Virol 85, 12201-12215, doi:10.1128/JVI.06048-11 (2011).

43 Janice Oh, H. S., Ken-En Gan, S Bertoletti, A., Tan, Y.J. T cell mediate protective immunity against emerging resperotory coronaviruses. Emerg Microbes Infect 1, 1-6 (2012).

44 Tseng, C. T. et al. Immunization with SARS coronavirus vaccines leads to pulmonary immunopathology on challenge with the SARS virus. PLoS One 7, e35421, doi:10.1371/journal.pone.0035421 (2012).

45 Kroemer, M. et al. COVID-19 patients display distinct SARS-CoV-2 specific T-cell responses according to disease severity. J Infect, 4816, doi:10.1016/j.jinf.2020.08.036 (2020).

46 Acero-Bedoya, S., Wozniak, P. S., Sanchez, P. J., Ramilo, O. \& Mejias, A. Recent Trends in RSV Immunoprophylaxis: Clinical Implications for the Infant. Am J Perinatol 36, S63-S67, doi:10.1055/s-0039-1691803 (2019).

47 Falloon, J. et al. An Adjuvanted, Postfusion F Protein-Based Vaccine Did Not Prevent Respiratory Syncytial Virus Illness in Older Adults. J Infect Dis 216, 1362-1370, doi:10.1093/infdis/jix503 (2017).

48 Mulligan, M. J. et al. Phase 1/2 study of COVID-19 RNA vaccine BNT162b1 in adults. Nature, doi:10.1038/s41586-020-2639-4 (2020).

49 Braun, E. \& Sauter, D. Furin-mediated protein processing in infectious diseases and cancer. Clin Transl Immunology 8, e1073, doi:10.1002/cti2.1073 (2019).

50 Nelson, C. S. et al. Human Cytomegalovirus Glycoprotein B Nucleoside-Modified mRNA Vaccine Elicits Antibody Responses with Greater Durability and Breadth than MF59-Adjuvanted gB Protein Immunization. J Virol 94, doi:10.1128/JVI.00186-20 (2020).

51 Seow, J., Graham, C., Merrick, B., Acors, S., Steel, K. J.A., Hemmings, O., O’Bryne, A., Kouphou, N., Pickering, S., Galao R. P., Betancor G., Wilson H.D., Signell A.W., Winstone H., Kerridge C., Temperton, N., Shell, L., Bisnauthsing, K., Moore, A., Green, A., Martiniz L., Stokes, B., Honey, J., Izquierdo-Barras, A., Arbane, G., Patel A., O'Connell, L., O'Hara, G., MacMahon, E., Douthwaite, S., Neibia, G., Batra, R., Martinez-Nunez, R., Edgeworth, J.D., Neil, S.J.D., Michael, H. Longitudinal evaluation and decline of Antibody responses in SARS-CoV-2 infection. doi:https://doi.org/10.1101/2020.07.09.20148429 (2020).

52 Wrapp, D. et al. Structural Basis for Potent Neutralization of Betacoronaviruses by SingleDomain Camelid Antibodies. Cell, doi:10.1016/j.cell.2020.04.031 (2020).

53 Rabaan, A. A. et al. SARS-CoV-2, SARS-CoV, and MERS-COV: A comparative overview. Infez Med 28, 174-184 (2020). 
54 DeRosa, F. et al. Improved Efficacy in a Fabry Disease Model Using a Systemic mRNA Liver Depot System as Compared to Enzyme Replacement Therapy. Mol Ther 27, 878-889,

599 doi:10.1016/j.ymthe.2019.03.001 (2019).

600

55 Sambrook, J. \& Russel, D. W. Molecular cloning. Laboratory manual. Third Edition edn, Vol. 1

601 (Cold Spring Harbor Laboratory Press, 2001).

602 